\title{
Data reduction for inverse modeling: an adaptive approach v1.0
}

\author{
Xiaoling Liu ${ }^{1}$, August L. Weinbren ${ }^{1}$, He Chang ${ }^{1}$, Jovan M. Tadić ${ }^{2}$, Marikate E. Mountain ${ }^{3}$, Michael E. Trudeau ${ }^{4}$, \\ Arlyn E. Andrews ${ }^{4}$, Zichong Chen ${ }^{1}$, and Scot M. Miller ${ }^{1}$ \\ ${ }^{1}$ Department of Environmental Health and Engineering, Johns Hopkins University, Baltimore, MD, USA \\ ${ }^{2}$ Climate and Ecosystem Sciences Division, Lawrence Berkeley National Laboratory, Berkeley, CA, USA \\ ${ }^{3}$ Atmospheric and Environmental Research, Inc., Lexington, MA, USA \\ ${ }^{4}$ Global Monitoring Laboratory, National Oceanic and Atmospheric Administration, Boulder, CO, USA
}

Correspondence: Scot M. Miller (smill191@jhu.edu)

Received: 24 July 2020 - Discussion started: 30 September 2020

Revised: 2 June 2021 - Accepted: 10 June 2021 - Published: 29 July 2021

\begin{abstract}
The number of greenhouse gas (GHG) observing satellites has greatly expanded in recent years, and these new datasets provide an unprecedented constraint on global GHG sources and sinks. However, a continuing challenge for inverse models that are used to estimate these sources and sinks is the sheer number of satellite observations, sometimes in the millions per day. These massive datasets often make it prohibitive to implement inverse modeling calculations and/or assimilate the observations using many types of atmospheric models. Although these satellite datasets are very large, the information content of any single observation is often modest and non-exclusive due to redundancy with neighboring observations and due to measurement noise. In this study, we develop an adaptive approach to reduce the size of satellite datasets using geostatistics. A guiding principle is to reduce the data more in regions with little variability in the observations and less in regions with high variability. We subsequently tune and evaluate the approach using synthetic and real data case studies for North America from NASA's Orbiting Carbon Observatory-2 (OCO-2) satellite. The proposed approach to data reduction yields more accurate $\mathrm{CO}_{2}$ flux estimates than the commonly used method of binning and averaging the satellite data. We further develop a metric for choosing a level of data reduction; we can reduce the satellite dataset to an average of one observation per $\sim 80-140 \mathrm{~km}$ for the specific case studies here without substantially compromising the flux estimate, but we find that reducing the data further quickly degrades the accuracy of the estimated fluxes. Overall, the approach developed here could be applied to a range of inverse problems that use very large trace gas datasets.
\end{abstract}

\section{Introduction}

Satellite observations of greenhouse gases (GHGs) have dramatically expanded over the past decade. New satellites with smaller footprints, wider viewing angles, and efficient scanning can collect millions of observations per day at high density and with broad spatial coverage. Remote sensing of carbon dioxide $\left(\mathrm{CO}_{2}\right)$ is a prime example. The Greenhouse Gases Observing Satellite (GOSAT), launched in early 2009, is the first satellite dedicated to observing $\mathrm{CO}_{2}$ and methane $\left(\mathrm{CH}_{4}\right)$ from space. GOSAT collects a modest $\sim 1 \times 10^{3}$ cloud-free soundings or observations per day. The Orbiting Carbon Observatory 2 (OCO-2), launched 5 years later in late 2014, is NASA's first satellite dedicated to observing $\mathrm{CO}_{2}$. It collects far more cloud-free soundings than GOSAT - on the order of $1 \times 10^{5}$ (Crisp, 2015; Eldering et al., 2017). By contrast, NASA's forthcoming Geostationary Carbon Cycle Observatory (GeoCarb), planned for launch in the early 2020 s, is slated to collect $\sim 1 \times 10^{7}$ soundings each day (Buis, 2018). A substantial fraction of these soundings will be unusable due to cloud contamination, but GeoCarb can reduce contamination by scanning cloud-free regions.

These satellites observe average $\mathrm{CO}_{2}$ mixing ratios across a vertical column of the atmosphere $\left(\mathrm{XCO}_{2}\right)$, and these $\mathrm{XCO}_{2}$ measurements can be used to estimate surface $\mathrm{CO}_{2}$ fluxes using inverse modeling. Specifically, an inverse model will combine satellite observations $(z$, dimensions $n \times 1)$ with an estimate of atmospheric transport $(\mathbf{H}, n \times m)$ to estimate surface fluxes $(\boldsymbol{s}, m \times 1)$ :

$z=\mathbf{H} s+\boldsymbol{\epsilon}$, 
where $\epsilon(n \times 1)$ is a vector of errors in the measurements and atmospheric modeling system. The objective of the inverse model is to estimate $\boldsymbol{s}$ given $\boldsymbol{z}$ and $\mathbf{H}$. Most existing inverse models also require an estimate of the statistical properties of $\epsilon$ to ensure that the solution does not under- or over-fit the atmospheric observations $(z)$. There are many different strategies for estimating the fluxes $(s)$; most existing studies implement an inverse model that is based upon Bayesian statistics. Refer to Rodgers (2000), Michalak et al. (2004), and Brasseur and Jacob (2017) for an overview of commonly used strategies for inverse modeling.

Large satellite datasets often pose computational problems for inverse modeling, specifically for calculations that involve the atmospheric model, H. The associated challenges vary depending upon the type of atmospheric model. For example, one approach to inverse modeling is to estimate $\mathbf{H}$ using a Lagrangian model, also known as a backtrajectory model. These models estimate how fluxes or emissions from different regions would impact mixing ratios at a downwind observation site. Commonly used models include the Stochastic Time-Inverted Lagrangian Transport (STILT) model (Lin et al., 2003; Nehrkorn et al., 2010), the FLEXible PARTicle dispersion model (FLEXPART) (Pisso et al., 2019), and the Hybrid Single-Particle Lagrangian Integrated Trajectory model (HYSPLIT) (Stein et al., 2016). One must run simulations of the back-trajectory model for each of the $n$ observations used in the inverse model, and each simulation becomes a different row of $\mathbf{H}$. As a result of this setup, the computational cost of the back-trajectory model scales with the number of observations, the size of the modeling domain, and the resolution of the model. This approach is commonly employed for ground- and aircraft-based atmospheric observations but can quickly become computationally challenging for large satellite datasets (e.g., Wu et al., 2018).

Another common approach to atmospheric modeling is to use a gridded atmospheric model, also known as an Eulerian model. Common models include the Goddard Earth Observing System - Chemistry model (GEOS-Chem) (e.g., Henze et al., 2007; Liu et al., 2017) and TM5 (e.g., Krol et al., 2005; Bergamaschi et al., 2005). These models are not typically used to explicitly calculate $\mathbf{H}$. Rather, these models are often used to calculate the product of $\mathbf{H}$ or $\mathbf{H}^{T}$ and a vector. One can then estimate $s$ by iterating toward the minimum of an objective function (e.g., Eq. S1 in the Supplement) using a series of matrix-vector products that involve $\mathbf{H}$ and $\mathbf{H}^{T}$ (e.g., Brasseur and Jacob, 2017). Large satellite datasets can also create computational challenges for inverse models that follow this approach. The model output must be interpolated to the locations and times of the observations and multiplied by satellite parameters like the pressure weighting function and averaging kernel. These calculations are often applied repeatedly during the course of iterative inverse modeling algorithms, and the computational cost of these calculations will increase with more observations. In addition, file input/output (I/O) can be a bottleneck for some types of at- mospheric models, and this cost increases as the number of observations increase. The GEOS-Chem model provides an illustrative example. In test simulations, the current GEOSChem forward and adjoint models for $\mathrm{CO}_{2}$ (v35, at the time of writing) required $\sim 30 \mathrm{~d}$ of wall-clock time to calculate the objective function and its gradient (i.e., first derivative) using a year of OCO-2 observations from the "lite" file (i.e., using $3.1 \times 10^{7}$ total observations for the year 2016; computed on the University of Minnesota Mesabi cluster using a global model spatial resolution of $2^{\circ}$ latitude by $2.5^{\circ}$ longitude). Most iterative inverse modeling algorithms require calculating this objective function and its gradient multiple times - at each iteration of the algorithm. By contrast, these same calculations required $\sim 0.5 \mathrm{~d}$ of wall-clock time using $10 \mathrm{~s}$ averages of the OCO-2 observations $\left(9 \times 10^{4}\right.$ total observations).

The most common solution to date for these computational problems is to reduce the size of the satellite dataset. One approach is to bin and average the data across a set interval and/or run the atmospheric model at a set interval along the satellite flight track. For example, recent inverse models for OCO-2 use data that have been binned and averaged every $10 \mathrm{~s}$ along the satellite flight track (e.g., Crowell et al., 2019). This approach yields approximately one observation per $70 \mathrm{~km}$, far fewer observations than the original OCO-2 dataset.

Relatedly, scientists that use a back-trajectory model for atmospheric simulations will often run the model at a set interval along the flight track. For example, scientists at NOAA have generated trajectory simulations for OCO-2 data over North America using STILT as part of the CarbonTrackerLagrange project (e.g., NOAA Global Monitoring Laboratory, 2020a; Miller et al., 2020). These runs have been generated for a single location every $2 \mathrm{~s}$ along each satellite flight track, thereby reducing the number of model simulations required. This $2 \mathrm{~s}$ interval yields just under one simulation per $10 \mathrm{~km}$, the spatial resolution of the meteorology fields used in the trajectory model simulations. The total computational cost of these simulations is substantial even using a dataset reduced to $2 \mathrm{~s}$ intervals; each STILT simulation (i.e., for a single observation location) requires $\sim 5 \mathrm{~h}$ of computing time on a single core of the NASA Pleiades supercomputer. NOAA scientists generated $\sim 9.88 \times 10^{4}$ STILT simulations for the year 2015 .

These existing strategies for data reduction present several challenges. First, one must decide how frequently to average the data (i.e., across how many seconds or kilometers) or how frequently to generate atmospheric simulations along a satellite flight track. It is not always practical to re-run the atmospheric model and inverse model with different levels of data reduction to decide on an optimal approach due to the computing time involved. Instead, this decision is often based upon the spatial resolution of the atmospheric transport model (e.g., NOAA Global Monitoring Laboratory, 2020a) or the anticipated spatial resolution of the flux estimate (e.g., 
Crowell et al., 2019). Second, one level of averaging or data reduction (e.g., 2 vs. $10 \mathrm{~s}$ ) may work better for one inverse modeling setup or one satellite dataset than another. Lastly, satellite observations are typically non-stationary: they exhibit different spatial and temporal variability in different locations and in different seasons (e.g., Katzfuss and Cressie, 2011; Hammerling et al., 2012a). These differences may be important to account for when reducing the size of the satellite dataset. For example, OCO-2 observations collected over the remote ocean have a lower variance and are correlated across longer distances than observations collected over terrestrial regions with heterogeneous surface sources and sinks (e.g., Eldering et al., 2017). A one-size-fits-all approach to data reduction may not be ideal in this circumstance. Instead, it may be advantageous to reduce the size of the dataset more in regions with little variability and less in regions with greater variability.

Scientists in other academic disciplines have also grappled with many of these challenges, albeit in the context of very different scientific applications. For example, data reduction has become common in computer graphics and data visualization because many remote sensing and/or medical images are too large to render and display at native resolution (e.g., $\mathrm{Li}$ et al., 2018). Numerous studies reduce the size of the image through a process known as mesh reduction; these algorithms reduce the mesh more in regions of the image with little variability and less in locations with high variability (e.g., Schroeder et al., 1992; Garland and Heckbert, 1997; Brodsky and Watson, 2000; Li et al., 2018). The algorithms are therefore also adaptable to different images.

Data reduction has also become common in weather data assimilation, in which the reduced datasets are typically referred to as "superobservations" or "superobs". In most existing meteorology studies, the data are divided into different grid boxes and averaged, analogous to the approach used in recent GHG studies (e.g., Lorenc, 1981; Miyoshi and Kunii, 2012). More recently, however, several studies have proposed "adaptive" or "intelligent" approaches to data reduction (e.g., Ochotta et al., 2005; Ramachandran et al., 2005; Lazarus et al., 2010; Richman et al., 2015). These studies preferentially reduce or thin the data more in regions where the observations have little variability or provide redundant information. Existing studies have used different algorithms to attain this goal, including mesh reduction (Ramachandran et al., 2005), data clustering (e.g., Ochotta et al., 2005), and machine learning (Richman et al., 2015). Compared to these meteorology studies, data reduction in atmospheric inverse modeling presents unique challenges. In weather data assimilation, observations are used to directly nudge or adjust a weather model in adjacent grid boxes. In inverse modeling, by contrast, the atmospheric observations and unknown GHG fluxes are fundamentally different quantities with complex relationships determined by atmospheric winds.

In the present study, we develop an approach to data reduction for inverse modeling of GHG observations. This ap- proach follows the principles of adaptive reduction: we reduce the $\mathrm{XCO}_{2}$ data more in regions with little variability in the observations and less in regions with high variability. The goals of this approach are two-fold. First, improve the computational feasibility of inverse modeling using satellite data while preserving the accuracy of the estimated fluxes. Second, develop an objective means to decide on the optimal level of data reduction for a given satellite dataset and a given inverse modeling problem. We subsequently tune and evaluate this approach using several case studies from the OCO-2 satellite - case studies that use synthetic and real data and case studies from different seasons of the year. We then compare $\mathrm{CO}_{2}$ fluxes estimated using the proposed approach against fluxes estimated using a satellite dataset that has been averaged to reduce its size. This comparison provides a lens to evaluate the costs and benefits of the proposed approach to data reduction vs. the commonly used approach of averaging the data. The approach described here is designed not only for OCO-2 but could be applied to current and future observations of $\mathrm{CO}_{2}$ (e.g., from GeoCarb) and observations of $\mathrm{CH}_{4}$ (e.g., from the TROPOspheric Monitoring Instrument, TROPOMI, and GeoCarb).

\section{Approach to data reduction}

We develop an approach to data reduction for inverse modeling that leverages tools from geostatistics. Geostatistical tools, like variogram modeling and kriging, have become widespread in spatial data analysis (e.g., Kitanidis, 1997; Wackernagel, 2003), and these tools are often straightforward to implement using software packages in R, MATLAB, Python, and other scientific programming languages. Furthermore, geostatistical tools are already used throughout inverse modeling and therefore offer an appealing framework for data reduction.

The overall strategy developed here is first to characterize the spatial properties of the observations using variogram analysis and second to use kriging to interpolate the satellite observations to a number of locations that are fewer than in the original dataset. The choice of locations is informed by the variogram analysis: we retain fewer locations in regions where the observations are correlated over longer distances and more locations in regions with a shorter decorrelation length.

\subsection{Step 1: evaluate the spatial properties of the satellite data}

We estimate the degree of spatial correlation in the satellite observations using a variogram analysis (e.g., Kitanidis, 1997). This analysis yields an estimate of the decorrelation length - the distance at which the correlation between any two observations is effectively zero. 
In this study, we estimate the decorrelation length by creating a variogram of the satellite observations (Fig. S6). A variogram is a geostatistical tool that is used to quantify the differences among observations as a function of distance. The variogram of the observations is often known as an empirical variogram, and we then fit a model to this empirical variogram using a least squares fit to estimate the decorrelation length (e.g., Kitanidis, 1997; Wackernagel, 2003). There are many possible choices for a variogram model, and we choose an exponential model with a nugget because it has been used in several existing studies of satellite-based $\mathrm{XCO}_{2}$ observations (e.g., Hammerling et al., 2012a; Zeng et al., 2014; Guo et al., 2015; Tadić et al., 2015, 2017). The covariances between observations in this model decay exponentially as a function of distance. Furthermore, the nugget component of the variogram model accounts for fine-scale variability and errors in the observations - specifically errors that are spatially uncorrelated. Note that the exponential model yields an estimate of the $e$-folding distance, the distance at which covariances decay by a factor of $e$. In this study, we report the decorrelation length $(l)$ or 3 times the $e$-folding distance; this is the distance at which the covariances effectively decay to zero. Refer to Kitanidis (1997) or Wackernagel (2003) for a review of different variogram models and model fitting.

We specifically estimate the decorrelation length along individual satellite flight tracks and estimate different lengths at different locations along each track. The spatial properties of the satellite observations often differ in different regions of the globe, and these differences are important to account for. In this particular study, we include all observations within $2000 \mathrm{~km}$ when making the estimate at each location along a flight track (as in Hammerling et al., 2012a, b). Note that we do not quantify correlations or covariances among different flight tracks or different days for the case studies using OCO-2 (Sect. 3); that satellite has a narrow swath of $\sim 10 \mathrm{~km}$ and a $16 \mathrm{~d}$ revisit time, so the individual flight tracks on a given day or week are spaced relatively far apart (e.g., Crisp, 2015; Eldering et al., 2017). For new and forthcoming satellites with a wider swath and/or more frequent revisit time, one could quantify zonal, meridional, and/or temporal decorrelation lengths, depending upon the characteristics of the satellite in question.

\subsection{Step 2: reduce the data using kriging}

We subsequently reduce the satellite dataset by estimating atmospheric $\mathrm{CO}_{2}$ at one location per fraction of a correlation length along each satellite flight track. For example, a modeler could reduce the dataset to one observation per $0.1 \mathrm{l}$ or $1.0 l$. The latter choice would reduce the size of the dataset to a much greater degree but increase the risk of losing information that would ultimately inform the inverse model. Sect. 2.3 discusses strategies for deciding on an optimal level of data reduction.
The correlation length will differ in different locations, and this procedure will therefore yield a different density of points in different regions. For example, the proposed approach will result in a greater density of points in regions where $\mathrm{XCO}_{2}$ varies across small spatial scales and a lower density of points in regions where $\mathrm{XCO}_{2}$ is correlated across long distances.

This approach is conceptually similar to several adaptive strategies for data reduction in other scientific fields. Many existing studies either remove, merge, or cluster data points based on spatial variability. In computer visualization, mesh reduction studies merge or remove vertices from the image based upon the curvature or flatness of the original image, and different studies use various metrics to quantify this curvature and flatness (e.g., Schroeder et al., 1992; Garland and Heckbert, 1997; Brodsky and Watson, 2000; Li et al., 2018). Studies in meteorology use similar algorithms. For example, Ochotta et al. (2005) developed a metric to cluster observations based upon both the squared distance between observations and the squared difference in the observation values. Similarly, Ramachandran et al. (2005) reduced the data based upon the variance of the data in each locale. In this particular study, we use the decorrelation length, a common tool in geostatistics, as a measure of variability in the original data and to guide the data reduction.

At each chosen location along the flight track, we subsequently interpolate the observations using ordinary kriging (e.g., Kitanidis, 1997). Numerous existing studies have applied various forms of kriging to interpolate satellite observations of $\mathrm{CO}_{2}$, including, for example, Katzfuss and Cressie (2011), Hammerling et al. (2012a), Hammerling et al. (2012b), and Tadić et al. (2015). Kriging accounts for the spatial and/or temporal properties of the quantity of interest, yielding a more accurate estimate. Kriging also yields an estimate of uncertainty in the estimated quantity - in this case uncertainties in the reduced $\mathrm{XCO}_{2}$ dataset. These uncertainties account for the variability (or lack thereof) in the OCO-2 data in the vicinity of each location, the density or sparsity of the original OCO-2 dataset near each location, and random noise in the OCO-2 observations, among other error sources. Both the best estimate of $\mathrm{XCO}_{2}$ and the corresponding uncertainties can be calculated using a simple linear system of equations.

We specifically implement ordinary kriging using a moving neighborhood (e.g., Kitanidis, 1997; Hammerling et al., $2012 a, b)$; the quantity of interest is only estimated at a single location at one time using nearby observations. This approach contrasts with other variants of kriging that incorporate all observations to estimate all unknown locations simultaneously. Ordinary kriging with a moving neighborhood is particularly useful when the observations are non-stationary and exhibit different spatial properties and/or error characteristics in different regions, as is often the case with $\mathrm{XCO}_{2}$ (e.g., Hammerling et al., 2012a). 
Ordinary kriging with a moving neighborhood requires two steps. First, a modeler must estimate the spatial properties of the observations in the vicinity of the estimation location. We estimate these properties as part of the analysis in Sect. 2.1 and use that estimate as an input in ordinary kriging. Second, we estimate $\mathrm{XCO}_{2}$ at each location of interest by solving a system of linear equations. Kitanidis (1997) describes this approach in detail, and Hammerling et al. (2012a, b) describe the application of moving neighborhood kriging to observations of $\mathrm{XCO}_{2}$. The $\mathrm{XCO}_{2}$ estimates from kriging can then be incorporated as observations in inverse modeling.

Note that traditional kriging models are designed to interpolate the quantity of interest to the same spatial support as the observations. In other words, the footprint size of the kriging estimate will be the same as that of the observations. This setup works well for the case study presented here; the atmospheric simulations in this study are generated using a back-trajectory model, and each simulation corresponds to a specific point location and time along an OCO-2 flight track. By contrast, a variant of kriging known as block kriging can be used to estimate a representative or average value for an entire grid box (e.g., Wackernagel, 2003; Tadić et al., 2015, 2017). This approach may be desirable when generating atmospheric simulations using an Eulerian model in which the outputs represent grid averages. Tadić et al. $(2015,2017)$ describe this approach in detail, including applications to interpolating $\mathrm{XCO}_{2}$.

\subsection{Step 3: decide on an optimal level of data reduction}

A modeler must decide on an optimal level of data reduction. That decision is often based on multiple considerations: the native spatial resolution of the atmospheric model, the computational demands of the inverse model, and the accuracy of the resulting flux estimate. For example, the resolution of the atmospheric model may help dictate a level of data reduction. An atmospheric model will not be able to resolve patterns in the fluxes at spatial scales smaller than the model resolution, so there may be little need to assimilate $\mathrm{CO}_{2}$ observations at a finer density than the model resolution.

For the specific algorithm described here, one must decide on a fraction of a correlation length and reduce the dataset accordingly. A modeler could decide on the optimal level of data reduction using a brute force approach: create numerous datasets with different levels of data reduction, run the inverse model on each, and decide on a level of data reduction based upon a comparison of the estimated fluxes. In practice, this approach is time consuming.

Instead, we propose a criterion for choosing a level of data reduction based upon the variance of the satellite data. We first select all data points in the original $\mathrm{CO}_{2}$ dataset that fall between two specific kriged points. We then calculate the variance of those selected points using the var() function in $\mathrm{R}$. We repeat this procedure for each pair of kriged points in the model domain. We finally average these variances calculated across each pair of kriged points. Some of this variance will undoubtedly be due to measurement error, but some of this variance will likely be due to real variability in atmospheric $\mathrm{CO}_{2}$.

This variance represents the variability in the data that is lost through the process of data reduction, and it provides a metric for choosing a level of data reduction that does not require re-running the inverse model. This number is smallest when the data reduction is minimal and increases for greater levels of data reduction. For the case studies in Sect. 3, this variance is often a nonlinear function of the level of data reduction; it increases slowly if the data reduction is minimal, reaches an inflection point, and then increases more quickly at greater levels of data reduction. A modeler can then choose a level of data reduction that is preferably below the inflection point and therefore reduces the potential for information loss while balancing the computational requirements of the inverse model.

We evaluate this proposed approach for deciding on a level of data reduction through several case studies based upon the OCO-2 satellite, described in detail in the next section (Sect. 3).

\section{Description of the case studies}

We evaluate the data reduction algorithm using three case studies based on the OCO-2 satellite. In each case, we estimate $\mathrm{CO}_{2}$ fluxes across North America for 6 weeks at a 3hourly temporal resolution and a $1^{\circ} \times 1^{\circ}$ latitude-longitude spatial resolution. Note that this setup targets a particular application of OCO-2 observations to inverse modeling across a continent. One could apply data reduction to inverse models that target urban areas or the entire globe, but the algorithm tuning (e.g., Sect. 2.3) and inverse modeling results will depend upon the particular application involved.

We specifically estimate fluxes using synthetic observations from July and early August 2015 using synthetic observations from March and early April 2015, and using real observations from July and early August 2015. Synthetic observations make it possible to compare the results against a known solution; they are therefore particularly useful for evaluating the data reduction algorithm proposed here. We further evaluate the algorithm in a real data simulation that mirrors real-world inverse modeling applications. Note that we present the details of the summer real data case study in the Supplement and focus on the synthetic case studies in the main text. The results of the real data case study are qualitatively very similar to the synthetic case studies, so we include that information in the Supplement to avoid duplicating similar information in the main text.

We further estimate the $\mathrm{CO}_{2}$ fluxes using a geostatistical inverse model (e.g., Kitanidis and Vomvoris, 1983; Michalak et al., 2004; Miller et al., 2020). The inverse model used here 
also has a non-informative prior. In other words, the prior has no spatial variability (e.g., Michalak et al., 2004; Mueller et al., 2008). As a result, any patterns in the estimated fluxes reflect the information content of the observations and not any prior information. This setup is identical to the case studies in Miller et al. (2020), and the reader is referred to both the Supplement and that study for additional details.

The case studies here also use atmospheric transport simulations from NOAA's CarbonTracker-Lagrange project (e.g., $\mathrm{Hu}$ et al., 2019; NOAA Global Monitoring Laboratory, 2020a). These simulations are used to create $H$ (Eq. 1) and are generated using the Weather Research and Forecasting (WRF) model coupled with the STILT model (e.g., Lin et al., 2003; Nehrkorn et al., 2010). The WRF simulations have a spatial resolution of $10 \mathrm{~km}$ over most of the continental United States and a resolution of $30 \mathrm{~km}$ across other regions of North America. The WRF-STILT simulations have also been processed to account for the pressure weighting function and averaging kernel of each OCO-2 observation. Miller et al. (2020) provides additional detail on the specific setup of the WRF-STILT runs used here. Note that the STILT simulations for CarbonTracker-Lagrange were generated every $2 \mathrm{~s}$ along the OCO-2 flight track and not at every individual OCO-2 observation due to the large number of observations and due to computational constraints. Hence, we only evaluate data reduction that yields fewer than one observation every $2 \mathrm{~s}$ for the case studies here.

We further create the synthetic data for each case study using WRF-STILT and $\mathrm{CO}_{2}$ fluxes from NOAA's CarbonTracker (CT2017) product (Peters et al., 2007; NOAA Global Monitoring Laboratory, 2020b). The synthetic $\mathrm{CO}_{2}$ fluxes not only include biospheric fluxes but also anthropogenic and biomass burning emissions. The synthetic observations also include noise $(\epsilon)$ that is added to simulate measurement and atmospheric modeling errors. For the summer case studies here, these errors have a variance of $(2 \mathrm{ppm})^{2}$ (as in Miller et al., 2020). We include error covariances to account for spatial correlation among these errors. We use the decorrelation length from Kulawik et al. (2019), who quantified errors in OCO-2 observations and estimated a decorrelation parameter of $0.3^{\circ}$ using an exponential variogram model. In the winter case study, we use a slightly smaller error variance of $(1.5 \mathrm{ppm})^{2}$ because there is less regional variability in atmospheric $\mathrm{CO}_{2}$ in winter. Note that we only include land nadir and land glint observations in the case studies and exclude ocean glint observations because those observations have known biases (O'Dell et al., 2018).

\section{Results and discussion}

\subsection{Spatial properties of the OCO-2 observations}

We estimate correlation lengths that are generally longer in winter when biospheric fluxes are small than in sum- mer when there are large spatial and temporal variations in biospheric fluxes. Figure 1 displays the estimated correlation lengths along the OCO-2 flight tracks for the summer (Fig. 1a) and winter (Fig. 1b) synthetic case studies. Most of the estimated correlation lengths range from $\sim 250$ to $1000 \mathrm{~km}$. Note that there are likely multiple different scales of variability in the OCO-2 observations: fine-scale variability due to retrieval errors (e.g., Kulawik et al., 2019; O'Dell et al., 2018), small-scale variability due to variations in mesoscale meteorology (e.g., Torres et al., 2019), and broad variability due to synoptic meteorology and regional patterns in $\mathrm{CO}_{2}$ fluxes. We specifically focus on quantifying synoptic-scale variability in Fig. 1 because the objective of the case studies is to estimate broad, regional patterns in $\mathrm{CO}_{2}$ fluxes across an entire continent.

The analysis in Fig. 1 also indicates substantial heterogeneity in the correlation lengths. Correlation lengths are often similar along a single flight track but vary among different tracks. These differences between flight tracks are most likely due to a combination of variations in synoptic meteorology and variability in the underlying $\mathrm{CO}_{2}$ fluxes. Indeed, several studies have shown that meteorological variability can explain a substantial fraction of variability in $\mathrm{XCO}_{2}$ across different spatial scales (e.g., Parazoo et al., 2008; Keppel-Aleks et al., 2011; Torres et al., 2019).

Two flight tracks that cross California, Oregon, and Washington illustrate the likely impacts of fluxes and meteorology on heterogeneity in the synthetic OCO-2 observations. One track, on 16 July 2015 , exhibits relatively little variability in $\mathrm{XCO}_{2}$, and we estimate an average correlation length of $794 \mathrm{~km}$ along the track with a standard deviation of $266 \mathrm{~km}$. By contrast, another nearby track from 21 July exhibits far more $\mathrm{XCO}_{2}$ variability, and we estimate a shorter mean correlation length of $221 \mathrm{~km}$ with a standard deviation of $53 \mathrm{~km}$ along the track. These large differences likely reflect differences in the underlying $\mathrm{CO}_{2}$ fluxes and in meteorology on the respective days. The 16 July track passes through eastern California, Nevada, eastern Oregon, and eastern Washington desert regions with little heterogeneity in $\mathrm{CO}_{2}$ fluxes. By contrast, the track from 21 July passes over the Sierra Nevada mountains and over multiple heterogeneous biome types (e.g., desert and temperate rainforest). Furthermore, weather maps indicate that a cold front passed through the Pacific Northwest on 21 July with variable winds on either side of the front (NOAA National Centers for Environmental Prediction Weather Prediction Center, 2020). These differences in transport and surface fluxes likely yield very different patterns of variability in two satellite flight tracks that are geographically close to one another. These results also imply that it is important to calculate correlation lengths for each individual flight track and not apply correlation length estimates from one track to another track or from one month/year to another month/year. Meteorology can easily vary from one day to another. Hence, we advise against pre-computing the 


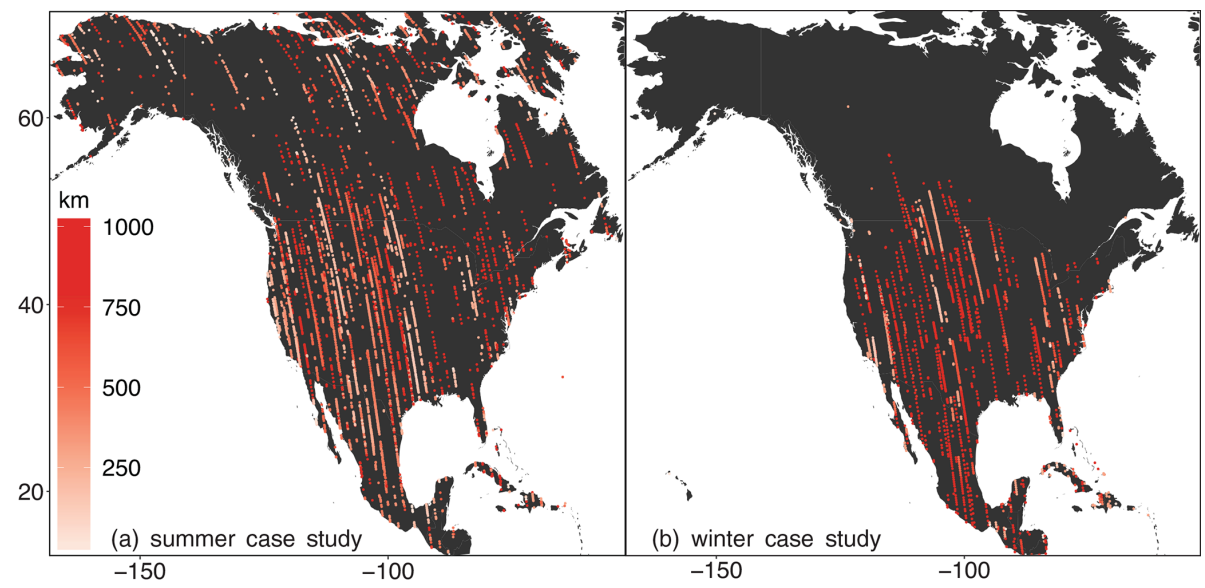

Figure 1. Correlation lengths estimated along OCO-2 flight tracks for (a) the summer synthetic case study and (b) the winter synthetic case study. The estimated correlation lengths are typically shorter in summer when biospheric fluxes exhibit high spatiotemporal variability and longer in winter when biospheric fluxes are small.

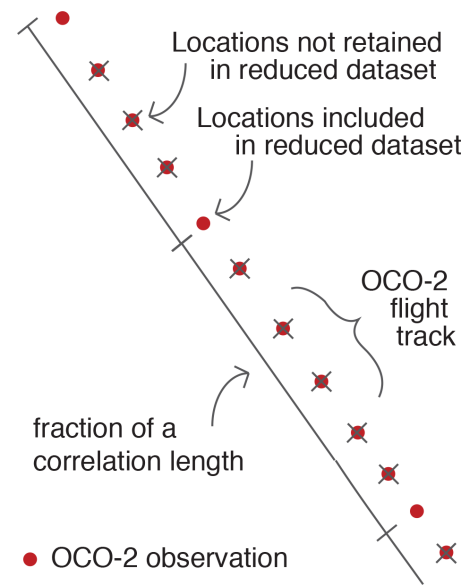

Figure 2. A schematic of the approach to data reduction proposed here. We estimate $\mathrm{XCO}_{2}$ at one location per fraction of a correlation length along the satellite flight track, where the specific fraction must be chosen by the user. We subsequently estimate $\mathrm{XCO}_{2}$ at each chosen location using ordinary kriging with a moving neighborhood.

correlation lengths for an individual track or an individual year and applying them to other tracks or other years.

\subsection{Estimated $\mathrm{CO}_{2}$ fluxes using the reduced $\mathrm{OCO}-2$ dataset}

The data reduction approach proposed here yields flux estimates that faithfully reproduce patterns in the synthetic $\mathrm{CO}_{2}$ observations. With that said, data reduction is always a compromise between the accuracy of the flux estimate and the computational requirements of the inverse model. As such, the accuracy of the flux estimate begins to degrade at high levels of data reduction. Figures 3 and 4 summarize many of these features and display maps of the time-averaged fluxes from the summer and winter case studies, respectively. The first panel (Figs. 3a and 4a) in each figure contains the CarbonTracker fluxes that were used to generate the synthetic OCO-2 observations. The second panel (Figs. $3 b$ and $4 b$ ) shows the fluxes estimated using the full, synthetic OCO-2 dataset with no reduction; the estimated $\mathrm{CO}_{2}$ fluxes shown in these panels do not have the same level of spatial definition as the original CarbonTracker fluxes (Figs. 3a and 4a), but the estimates broadly reproduce the spatial patterns in CarbonTracker. The inverse model in this study uses a noninformative prior, so any patterns in panel (b) are solely informed by the observations and not the result of prior flux information. The patterns in Figs. $3 \mathrm{~b}$ and $4 \mathrm{~b}$ indicate that the synthetic OCO-2 observations can be used to recover continental-scale spatial features in the fluxes, but the observations and inverse model do not have the sensitivity or information content to recover more detailed features. Subsequent panels (Figs. 3c and 4c) display the fluxes estimated using observations that have been reduced to a modest level one observation per $0.2 l$ or an average of one observation per $100 \mathrm{~km}$ for the summer case study and $140 \mathrm{~km}$ for the winter case study. The final panel in each figure displays a severe level of reduction - one observation per $0.75 l$, an average of one observation per $400 \mathrm{~km}$ for the summer case study and $540 \mathrm{~km}$ for the winter case study. In both the summer and winter case studies, the modest level of data reduction (one observation per $0.2 l$ ) reduces the total number of observations by $\sim 70 \%$ and the severe data reduction (one observation per $0.75 l$ ) by $\sim 90 \%$. A data reduction of $70 \%$ or more and the corresponding reduction in the number of STILT simulations would yield substantial computational savings given the large computational cost required associated with STILT (Sect. 1). 


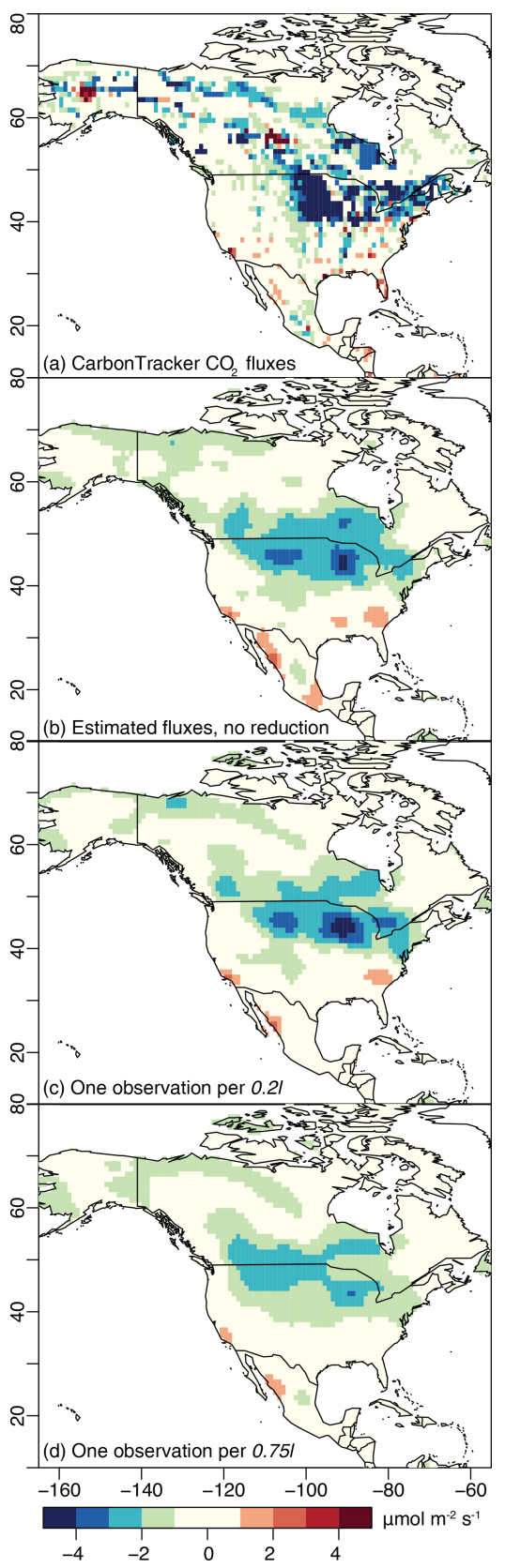

Figure 3. $\mathrm{CO}_{2}$ fluxes estimated for the summer 2015 synthetic case study, averaged across the 6-week study window: (a) the synthetic $\mathrm{CO}_{2}$ fluxes from NOAA's CarbonTracker estimate, (b) fluxes estimated from $\mathrm{XCO}_{2}$ data with no reduction (6799 data points), (c) fluxes estimated from data reduced to one point per $0.2 l$ (2263 data points), and (d) fluxes estimated from data reduced to one point per $0.75 l$ ( 755 data points). The estimate with no reduction (b) and a reduction of $0.2 l$ (c) reproduce broad, continental-scale spatial patterns in the synthetic fluxes (a), while the estimate with $0.75 l$ reduction has lost spatial definition. Note that the inverse model here uses a non-informative prior, so any patterns in the flux estimates are informed by the observations and not by any prior flux information.
In each case, the fluxes using the $0.2 l$ data (Figs. $3 c$ and $4 c$ ) reproduce spatial patterns in the fluxes estimated with no reduction (Figs. 3b and 4b). By contrast, the fluxes estimated using the $0.75 l$ data lack spatial definition and are therefore not an ideal estimate of the synthetic fluxes (Figs. 3a and 4a). Note that we also conducted a real data case study for summer 2015. Those results have broadly similar characteristics to the synthetic data case study and are discussed in detail in the Supplement.

In both of the case studies, the data reduction approach proposed here yields more accurate flux estimates than binning and averaging the observations. We reduce the data using both averaging and the geostatistical approach proposed in this study. We subsequently estimate fluxes using the reduced datasets and compare the results against the fluxes estimated using the full dataset without any data reduction. Figure 5 displays the results of this analysis: the root mean squared error (RMSE) of the grid-scale and 3-hourly estimated fluxes relative to fluxes calculated from the full dataset. In both the winter and summer case studies and at almost all levels of data reduction, the geostatistical approach produces fluxes with a lower RMSE.

Note that all results in Fig. 5 display a clear inflection point: the RMSE is relatively low at low levels of data reduction and increases rapidly at high levels of reduction. The chosen level of data reduction should be at or below this inflection point or else the inverse model will yield an inaccurate flux estimate. The inflection point for the geostatistical approach occurs at a higher level of data reduction than for data averaging. In other words, the RMSE for the geostatistical approach remains low at a greater degree of data reduction than for averaging.

The RMSE, however, may not be the only criterion to consider when deciding on a level of data reduction. Specifically, the spatial patterns in the fluxes begin to degrade at a lower level of data reduction than the RMSE. For example, in both the winter, summer, and real data case studies, the monthly averaged fluxes begin to lose spatial definition at data reduction levels greater than $0.15 l$ to $0.2 l$ (an average of one observation per $80-100 \mathrm{~km}$ for the summer case study and one observation per $100-140 \mathrm{~km}$ for the winter case study). Hence, it may be advisable to balance multiple criteria when deciding on an optimal level of data reduction depending upon the goals of the inverse modeling study.

\subsection{Determining an optimal level of data reduction}

In the previous section (Sect. 4.2), we evaluate the data reduction by comparing the resulting estimates of $\mathrm{CO}_{2}$ fluxes. However, this approach may not work well if the inverse model is time-consuming and/or computationally intensive, as is often the case for satellite-based inverse modeling. For example, in Fig. 5 we run the inverse model 20 times for each case study to estimate the fluxes using different levels of data reduction and evaluate the results against fluxes estimated us- 


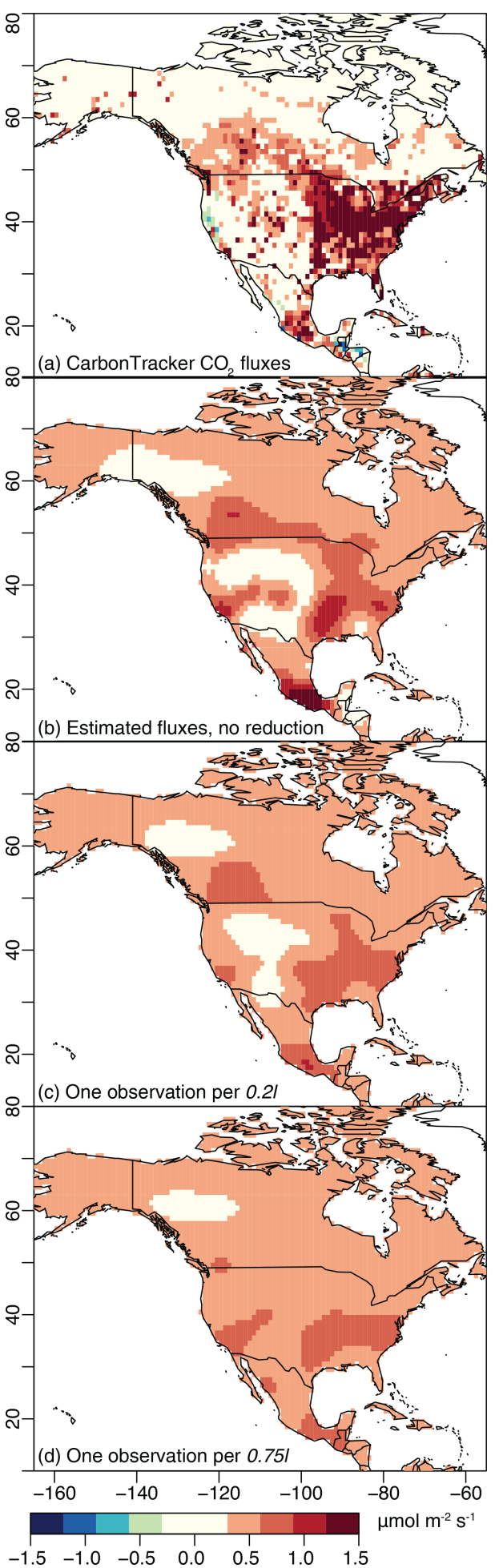

Figure 4. $\mathrm{CO}_{2}$ fluxes estimated for the winter case study, analogous to Fig. 3. The fluxes estimated with modest data reduction (c; 1098 data points) reproduce the patterns in the flux estimate with no reduction (b; 4183 data points). By contrast, large data reduction (d; 251 data points) yields fluxes with little spatial variability. ing the original, synthetic OCO-2 data. An inverse model can take days to run using large satellite datasets, so it may not be feasible or desirable to run the inverse model numerous times. Furthermore, one may want to decide on an optimal level of data reduction before running back-trajectory simulations using a model like STILT. We therefore propose an approach to evaluate the data reduction in a way that does not require running the inverse model or an atmospheric model like STILT (Sect. 2.3). This approach is based upon the variance of the original data between each of the reduced data points; this variance is a measure of the variability in the data that is lost through the process of data reduction.

In each of the OCO-2 case studies, this metric provides a reasonable and informative means to decide on an appropriate level of data reduction. Figure 6 displays this metric calculated for the summer (panel a) and winter (panel b) case studies at multiple different levels of data reduction. The variance lost through the process of data reduction is lowest at small levels of data reduction and increases nonlinearly at higher levels of data reduction. The summer case study (panel a) is highly nonlinear and reaches a very clear inflection point. By contrast, the winter case study (panel b) does not have as clear of an inflection point, but the variance does increase more quickly at higher levels of data reduction.

This metric also mirrors many of the patterns in the flux maps (Figs. 3 and 4) and RMSE calculations (Fig. 5). For example, fluxes in the summer case study lose spatial definition at data reduction levels greater than $0.2 l$ (equivalent to 2263 data points). Indeed, the variances in Fig. 6a begin to increase more rapidly after data reduction levels greater than $0.2 l$. By contrast, the fluxes in the winter case study progressively lose spatial definition, but that loss is particularly notable at high levels of reduction. That pattern is similar to the pattern in the variances in Fig. 6b. Furthermore, the patterns in Fig. 6 also mirror many of the patterns in the RMSE (Fig. 5). Both the RMSE and variances for the summer case study reach an inflection point between 2000 and 1000 observations, at which point both begin to increase rapidly. The RMSE and variance plots for the winter case study do not look identical (Figs. 5b and 6b). With that said, the pattern in the winter case study looks qualitatively more akin to the degradation of spatial patterns in the plotted fluxes than it does to the RMSE in Fig. 5b.

At the end of the day, it is arguably difficult to identify a single metric for deciding on an appropriate level of data reduction, and the right metric may depend upon the goal of the inverse model (e.g., identifying spatial patterns, temporal patterns, and/or flux totals). With that said, the metric proposed in this section is a computationally efficient option that summarizes many of the features of data reduction described in the previous sections (e.g., Sect. 4.2). 


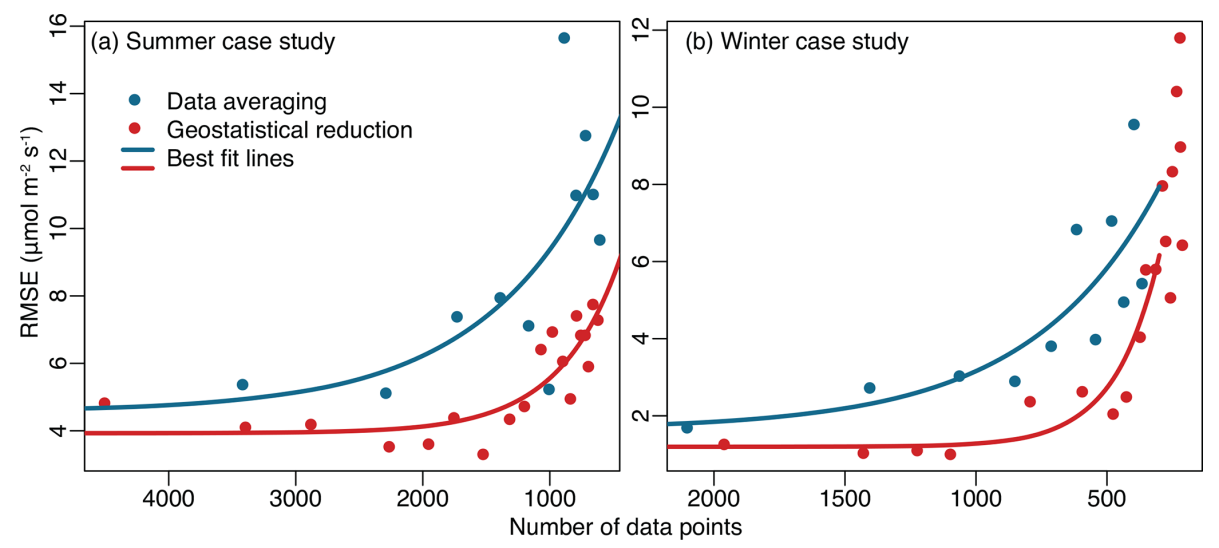

Figure 5. Root mean squared error (RMSE) of the fluxes estimated using data reduction relative to the fluxes estimated without data reduction. The figure displays results for the summer (a) and winter (b) case studies. Fluxes estimated using the data reduction approach proposed here have a lower RMSE relative to those estimated using the binning and averaging approach to data reduction.

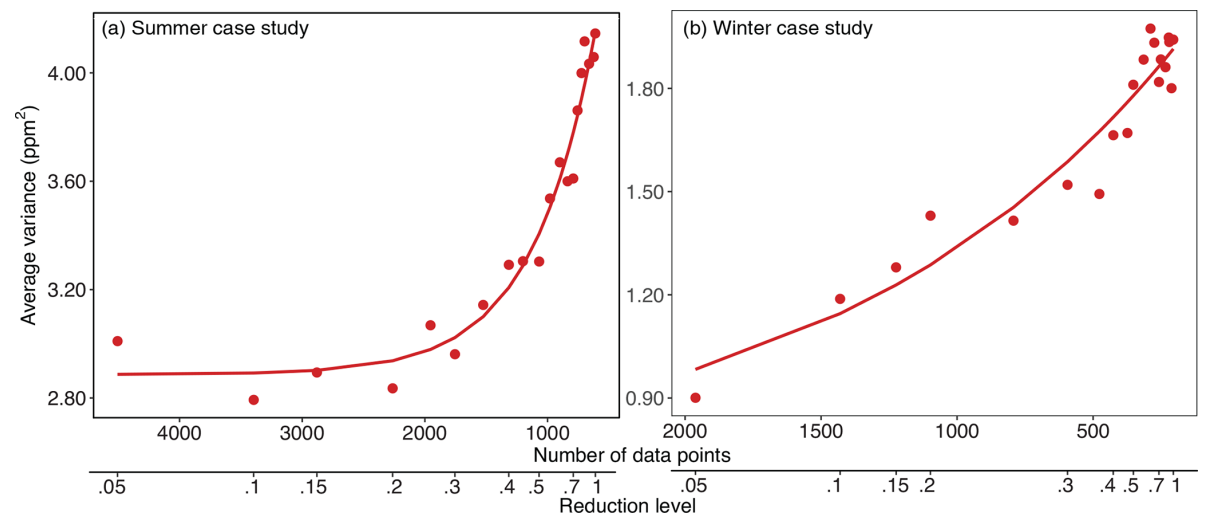

Figure 6. The amount of variance in the data that is lost through the process of data reduction for the summer (a) and winter (b) case studies. These plots provide a metric to help decide on an appropriate level of reduction and do not require costly runs of the inverse model to be generated.

\subsection{Computational costs}

We find that the computational costs of the data reduction algorithm are reasonable for the case studies explored here and are far less than the computing time associated with generating atmospheric model simulations. Both the variogram analysis (Sect. 2.1) and kriging (Sect. 2.2) are implemented using a moving neighborhood, thereby limiting the number of observations included in any given variogram or kriging calculation and reducing computing time. For example, we use a moving neighborhood with a radius of $2000 \mathrm{~km}$ for the case studies (as in Hammerling et al., 2012a), approximately half the width of the continental United States. Each empirical variogram required an average of $0.05 \mathrm{~s}$ to calculate using the $\mathrm{R}$ programming language, and each kriging estimate required an average of $0.02 \mathrm{~s}$. By contrast, a single STILT model simulation corresponding to a single OCO-2 observation required far more computing time (see Sect. 1).
Furthermore, one can distribute the variogram and kriging calculations across multiple computing cores and nodes, reducing the required computing time. Specifically, the calculation of each decorrelation length and each kriging estimate is independent of every other calculation or location, so these individual calculations can be spread across as many cores or nodes as desired. There is also some flexibility in the implementation of this algorithm and therefore in its computational cost. We calculate the variogram every $2 \mathrm{~s}$ along the OCO-2 flight track, but one could calculate the variogram at lessfrequent intervals. For example, Hammerling et al. (2012a) implement moving neighborhood kriging for synthetic OCO2 observations and calculate variogram parameters for each location on a $1^{\circ}$ latitude by $1.25^{\circ}$ longitude grid. One can also define the moving neighborhood differently with computational considerations in mind. For example, Tadić et al. $(2015,2017)$ limit the moving neighborhood to $500 \mathrm{XCO}_{2}$ observations. Instead of including all observations within a given radius, they choose observations to include in the mov- 
ing neighborhood using a randomized algorithm, and the algorithm preferentially chooses observations that are closer to the estimation location over observations that are far away. That strategy yields accurate variogram parameters and kriging estimates while ensuring that the number of observations within a moving neighborhood is not so large as to pose a computational burden.

\section{Conclusions}

In many instances, new satellite datasets are simply too large to assimilate in an inverse model given the current computational limitations of existing atmospheric models. In an ideal world, it would be possible to assimilate all available GHG observations to exploit the full information content of these massive new satellite datasets. However, that ideal is not computationally feasible in many instances, and modelers often need a strategy to reduce the size of these datasets. At minimum, this strategy should reduce the computational demands of inverse modeling while yielding flux estimates that accurately reproduce key information on the magnitude and distribution of surface fluxes. A complicating factor is that satellite observations often exhibit very different variability in different regions and/or on different days, depending on factors like regional variability in GHG fluxes and variations in meteorology. In this work, we argue that a data reduction strategy should account for this variability and that doing so typically yields a more accurate flux estimate.

One could develop a strategy for data reduction using many different statistical and mathematical tools, and we specifically develop a strategy using geostatistics because it provides a convenient way to quantify and account for the spatial variability in the satellite observations. In the case studies presented here based on NASA's OCO-2 satellite, this strategy outperforms data averaging, a common and straightforward approach to data reduction but one that does not account for the variable spatial properties of the observations. The specific implementation of this strategy will likely vary depending upon the satellite dataset in question and the specifics of the atmospheric model. To that end, we also develop and evaluate a computationally efficient metric to help choose an appropriate level of data reduction - a metric that does not require re-running the inverse model numerous times.

Future computational improvements to atmospheric models and increased access to high performance computing resources will hopefully make it possible to implement inverse modeling with larger and larger atmospheric datasets while minimizing the need for data reduction. With that said, forthcoming satellites like NASA's GeoCarb mission promise to collect unprecedented numbers of atmospheric GHG observations, and these new missions may make data reduction more necessary than ever.
Code and data availability. The code used in this study for data reduction is available on Zenodo at https://doi.org/10.5281/zenodo.3899317 (Liu et al., 2020). The inverse modeling code used in the case studies is also available at https://doi.org/10.5281/zenodo.3595574 (Miller, 2019), and the model simulations used to construct the summer case study are available on Zenodo at https://doi.org/10.5281/zenodo.3241467 (Miller et al., 2019).

Supplement. The supplement related to this article is available online at: https://doi.org/10.5194/gmd-14-4683-2021-supplement.

Author contributions. XL, ALW, JT, and SMM designed the study. $\mathrm{XL}, \mathrm{ALW}, \mathrm{HC}, \mathrm{ZC}$, and SMM conducted the analysis. MM, MT, and AA developed the strategy for simulating OCO-2 soundings using WRF-STILT and provided the footprints. SMM drafted the manuscript, and all authors helped edit the manuscript.

Competing interests. The authors declare that they have no conflict of interest.

Disclaimer. Publisher's note: Copernicus Publications remains neutral with regard to jurisdictional claims in published maps and institutional affiliations.

Acknowledgements. We thank Thomas Nehrkorn (Atmospheric and Environmental Research, AER, Inc.) for help with the atmospheric modeling simulations used in this study. We also thank Amy Braverman (NASA) for her advice and input on the project.

Financial support. This research has been supported by NASA ROSES (grant no. 80NSSC18K0976). CarbonTracker-Lagrange footprint production was supported by the NASA Carbon Monitoring System via interagency agreement NNH14AY37I.

Review statement. This paper was edited by Havala Pye and reviewed by two anonymous referees.

\section{References}

Bergamaschi, P., Krol, M., Dentener, F., Vermeulen, A., Meinhardt, F., Graul, R., Ramonet, M., Peters, W., and Dlugokencky, E. J.: Inverse modelling of national and European $\mathrm{CH}_{4}$ emissions using the atmospheric zoom model TM5, Atmos. Chem. Phys., 5, 2431-2460, https://doi.org/10.5194/acp-5-2431-2005, 2005.

Brasseur, G. and Jacob, D.: Modeling of Atmospheric Chemistry, Cambridge University Press, Cambridge, 2017.

Brodsky, D. and Watson, B.: Model simplification through refinement, in: Proceedings of Graphics Interface 2000: Montréal, Québec, Canada, 15-17 May 2000. 
Buis, A.: GeoCarb: A New View of Carbon Over the Americas, ExploreEarth, available at: https://www.nasa.gov/feature/jpl/ geocarb-a-new-view-of-carbon-over-the-americas (last access: 17 July 2020), 2018.

Crisp, D.: Measuring atmospheric carbon dioxide from space with the Orbiting Carbon Observatory-2 (OCO-2), in: Earth Observing Systems XX, vol. 9607, edited by Butler, J. J., Xiong, X. J., and $\mathrm{Gu}, \mathrm{X}$., International Society for Optics and Photonics, SPIE, https://doi.org/10.1117/12.2187291, 1-7, 2015.

Crowell, S., Baker, D., Schuh, A., Basu, S., Jacobson, A. R., Chevallier, F., Liu, J., Deng, F., Feng, L., McKain, K., Chatterjee, A., Miller, J. B., Stephens, B. B., Eldering, A., Crisp, D., Schimel, D., Nassar, R., O’Dell, C. W., Oda, T., Sweeney, C., Palmer, P. I., and Jones, D. B. A.: The 2015-2016 carbon cycle as seen from OCO-2 and the global in situ network, Atmos. Chem. Phys., 19, 9797-9831, https://doi.org/10.5194/acp-19-9797-2019, 2019.

Eldering, A., Wennberg, P., Crisp, D., Schimel, D., Gunson, M., Chatterjee, A., Liu, J., Schwandner, F., Sun, Y., O'Dell, C. W., Frankenberg, C., Taylor, T., Fisher, B., Osterman, G. B., Wunch, D., Hakkarainen, J., Tamminen, J., and Weir, B.: The Orbiting Carbon Observatory-2 early science investigations of regional carbon dioxide fluxes, Science, 358, eaam5745, https://doi.org/10.1126/science.aam5745, 2017.

Garland, M. and Heckbert, P. S.: Surface simplification using quadric error metrics, in: SIGGRAPH '97: Proceedings of the 24th annual conference on Computer graphics and interactive techniques, Los Angeles California USA on 3-8 August, 209216, https://doi.org/10.1145/258734.258849, 1997.

Guo, L., Lei, L., Zeng, Z., Zou, P., Liu, D., and Zhang, B.: Evaluation of Spatio-Temporal Variogram Models for Mapping $\mathrm{XCO}_{2}$ Using Satellite Observations: A Case Study in China, IEEE J. Sel. Top. Appl., 8, 376-385, https://doi.org/10.1109/JSTARS.2014.2363019, 2015.

Hammerling, D. M., Michalak, A. M., and Kawa, S. R.: Mapping of $\mathrm{CO}_{2}$ at high spatiotemporal resolution using satellite observations: Global distributions from OCO-2, J. Geophys. Res.Atmos., 117, D06306, https://doi.org/10.1029/2011JD017015, 2012a.

Hammerling, D. M., Michalak, A. M., O'Dell, C., and Kawa, S. R.: Global $\mathrm{CO}_{2}$ distributions over land from the Greenhouse Gases Observing Satellite (GOSAT), Geophys. Res. Lett., 39, L08804, https://doi.org/10.1029/2012GL051203, 2012b.

Henze, D. K., Hakami, A., and Seinfeld, J. H.: Development of the adjoint of GEOS-Chem, Atmos. Chem. Phys., 7, 2413-2433, https://doi.org/10.5194/acp-7-2413-2007, 2007.

Hu, L., Andrews, A. E., Thoning, K. W., Sweeney, C., Miller, J. B., Michalak, A. M., Dlugokencky, E., Tans, P. P., Shiga, Y. P., Mountain, M., Nehrkorn, T., Montzka, S. A., McKain, K., Kofler, J., Trudeau, M., Michel, S. E., Biraud, S. C., Fischer, M. L., Worthy, D. E. J., Vaughn, B. H., White, J. W. C., Yadav, V., Basu, S., and van der Velde, I. R.: Enhanced North American carbon uptake associated with El Niño, Science Advances, 5, eaaw0076, https://doi.org/10.1126/sciadv.aaw0076, 2019.

Katzfuss, M. and Cressie, N.: Tutorial on fixed rank kriging (FRK) of $\mathrm{CO}_{2}$ data, Tech. Rep. 858, Department of Statistics, The Ohio State University, Columbus, Ohio, available at: https://niasra.uow.edu.au/content/groups/public/@web/@inf/ $@$ math/documents/mm/uow175999.pdf (last access: 17 July 2020), 2011.
Keppel-Aleks, G., Wennberg, P. O., and Schneider, T.: Sources of variations in total column carbon dioxide, Atmos. Chem. Phys., 11, 3581-3593, https://doi.org/10.5194/acp-11-35812011, 2011.

Kitanidis, P.: Introduction to Geostatistics: Applications in Hydrogeology, Stanford-Cambridge program, Cambridge University Press, Cambridge, 1997.

Kitanidis, P. K. and Vomvoris, E. G.: A geostatistical approach to the inverse problem in groundwater modeling (steady state) and one-dimensional simulations, Water Resour. Res., 19, 677-690, https://doi.org/10.1029/WR019i003p00677, 1983.

Krol, M., Houweling, S., Bregman, B., van den Broek, M., Segers, A., van Velthoven, P., Peters, W., Dentener, F., and Bergamaschi, P.: The two-way nested global chemistry-transport zoom model TM5: algorithm and applications, Atmos. Chem. Phys., 5, 417432, https://doi.org/10.5194/acp-5-417-2005, 2005.

Kulawik, S. S., Crowell, S., Baker, D., Liu, J., McKain, K., Sweeney, C., Biraud, S. C., Wofsy, S., O’Dell, C. W., Wennberg, P. O., Wunch, D., Roehl, C. M., Deutscher, N. M., Kiel, M., Griffith, D. W. T., Velazco, V. A., Notholt, J., Warneke, T., Petri, C., De Mazière, M., Sha, M. K., Sussmann, R., Rettinger, M., Pollard, D. F., Morino, I., Uchino, O., Hase, F., Feist, D. G., Roche, S., Strong, K., Kivi, R., Iraci, L., Shiomi, K., Dubey, M. K., Sepulveda, E., Rodriguez, O. E. G., Té, Y., Jeseck, P., Heikkinen, P., Dlugokencky, E. J., Gunson, M. R., Eldering, A., Crisp, D., Fisher, B., and Osterman, G. B.: Characterization of OCO-2 and ACOS-GOSAT biases and errors for $\mathrm{CO}_{2}$ flux estimates, Atmos. Meas. Tech. Discuss. [preprint], https://doi.org/10.5194/amt-2019-257, 2019.

Lazarus, S. M., Splitt, M. E., Lueken, M. D., Ramachandran, R., Li, X., Movva, S., Graves, S. J., and Zavodsky, B. T.: Evaluation of Data Reduction Algorithms for Real-Time Analysis, Weather Forecast., 25, 837-851, https://doi.org/10.1175/2010WAF2222296.1, 2010.

Li, S., Marsaglia, N., Garth, C., Woodring, J., Clyne, J., and Childs, H.: Data Reduction Techniques for Simulation, Visualization and Data Analysis, Comput. Graph. Forum, 37, 422-447, https://doi.org/10.1111/cgf.13336, 2018.

Lin, J. C., Gerbig, C., Wofsy, S. C., Andrews, A. E., Daube, B. C., Davis, K. J., and Grainger, C. A.: A near-field tool for simulating the upstream influence of atmospheric observations: The Stochastic Time-Inverted Lagrangian Transport (STILT) model, J. Geophys. Res.-Atmos., 108, 4493, https://doi.org/10.1029/2002JD003161, 2003.

Liu, J., Bowman, K. W., Schimel, D. S., Parazoo, N. C., Jiang, Z., Lee, M., Bloom, A. A., Wunch, D., Frankenberg, C., Sun, Y., O’Dell, C. W., Gurney, K. R., Menemenlis, D., Gierach, M., Crisp, D., and Eldering, A.: Contrasting carbon cycle responses of the tropical continents to the 2015-2016 El Niño, Science, 358, eaam5690, https://doi.org/10.1126/science.aam5690, 2017.

Liu, X., Miller, S. M., and Weinbren, A.: greenhousegaslab/data_reduction: data reduction for large atmospheric satellite datasets (Version v1.0.0), Zenodo [code], https://doi.org/10.5281/zenodo.3899317, 2020.

Lorenc, A. C.: A Global Three-Dimensional Multivariate Statistical Interpolation Scheme, Mon. Weather Rev., 109, 701-721, https://doi.org/10.1175/15200493(1981)109<0701:AGTDMS>2.0.CO;2, 1981. 
Michalak, A. M., Bruhwiler, L., and Tans, P. P.: A geostatistical approach to surface flux estimation of atmospheric trace gases, J. Geophys. Res.-Atmos., 109, D14109, https://doi.org/10.1029/2003JD004422, 2004.

Miller, S. M.: greenhousegaslab/geostatistical_inverse_modeling: Geostatistical inverse modeling with large atmospheric datasets (Version v1.1), Zenodo [code], https://doi.org/10.5281/zenodo.3595574, 2019.

Miller, S. M., Saibaba, A. K., Trudeau, M. E., Andrews, A. E., Nehrkorn, T., and Mountain, M. E.: Geostatistical inverse modeling with large atmospheric data: data files for a case study from OCO-2 (Version 1.0), Zenodo [data set], https://doi.org/10.5281/zenodo.3241467, 2019.

Miller, S. M., Saibaba, A. K., Trudeau, M. E., Mountain, M. E., and Andrews, A. E.: Geostatistical inverse modeling with very large datasets: an example from the Orbiting Carbon Observatory 2 (OCO-2) satellite, Geosci. Model Dev., 13, 1771-1785, https://doi.org/10.5194/gmd-13-1771-2020, 2020.

Miyoshi, T. and Kunii, M.: Using AIRS retrievals in the WRF-LETKF system to improve regional numerical weather prediction, Tellus A, 64, 18408, https://doi.org/10.3402/tellusa.v64i0.18408, 2012.

Mueller, K. L., Gourdji, S. M., and Michalak, A. M.: Global monthly averaged $\mathrm{CO}_{2}$ fluxes recovered using a geostatistical inverse modeling approach: 1. Results using atmospheric measurements, J. Geophys. Res.-Atmos., 113, D21114, https://doi.org/10.1029/2007JD009734, 2008.

Nehrkorn, T., Eluszkiewicz, J., Wofsy, S. C., Lin, J. C., Gerbig, C., Longo, M., and Freitas, S.: Coupled weather research and forecasting-stochastic time-inverted lagrangian transport (WRF-STILT) model, Meteorol. Atmos. Phys., 107, 51-64, https://doi.org/10.1007/s00703-010-0068-x, 2010.

NOAA Global Monitoring Laboratory: CarbonTracker - Lagrange, available at: https://www.esrl.noaa.gov/gmd/ccgg/ carbontracker-lagrange/ (last access: 17 July 2020), 2020a.

NOAA Global Monitoring Laboratory: CarbonTracker, available at: https://www.esrl.noaa.gov/gmd/ccgg/carbontracker/ (last access: 17 July 2020), 2020b.

NOAA National Centers for Environmental Prediction Weather Prediction Center: Daily Weather Map, available at: https://www. wpc.ncep.noaa.gov/dailywxmap/ (last access: 17 July 2020), 2020.

Ochotta, T., Gebhardt, C., Saupe, D., and Wergen, W.: Adaptive thinning of atmospheric observations in data assimilation with vector quantization and filtering methods, Q. J. Roy. Meteor. Soc., 131, 3427-3437, https://doi.org/10.1256/qj.05.94, 2005.

O’Dell, C. W., Eldering, A., Wennberg, P. O., Crisp, D., Gunson, M. R., Fisher, B., Frankenberg, C., Kiel, M., Lindqvist, H., Mandrake, L., Merrelli, A., Natraj, V., Nelson, R. R., Osterman, G. B., Payne, V. H., Taylor, T. E., Wunch, D., Drouin, B. J., Oyafuso, F., Chang, A., McDuffie, J., Smyth, M., Baker, D. F., Basu, S., Chevallier, F., Crowell, S. M. R., Feng, L., Palmer, P. I., Dubey, M., García, O. E., Griffith, D. W. T., Hase, F., Iraci, L. T., Kivi, R., Morino, I., Notholt, J., Ohyama, H., Petri, C., Roehl, C. M., Sha, M. K., Strong, K., Sussmann, R., Te, Y., Uchino, O., and Velazco, V. A.: Improved retrievals of carbon dioxide from Orbiting Carbon Observatory-2 with the version 8 ACOS algorithm, Atmos. Meas. Tech., 11, 6539-6576, https://doi.org/10.5194/amt11-6539-2018, 2018.
Parazoo, N. C., Denning, A. S., Kawa, S. R., Corbin, K. D., Lokupitiya, R. S., and Baker, I. T.: Mechanisms for synoptic variations of atmospheric $\mathrm{CO}_{2}$ in North America, South America and Europe, Atmos. Chem. Phys., 8, 7239-7254, https://doi.org/10.5194/acp-8-7239-2008, 2008.

Peters, W., Jacobson, A. R., Sweeney, C., Andrews, A. E., Conway, T. J., Masarie, K., Miller, J. B., Bruhwiler, L. M. P., Pétron, G., Hirsch, A. I., Worthy, D. E. J., van der Werf, G. R., Randerson, J. T., Wennberg, P. O., Krol, M. C., and Tans, P. P.: An atmospheric perspective on North American carbon dioxide exchange: CarbonTracker, P. Natl. Acad. Sci. USA, 104, 1892518930, https://doi.org/10.1073/pnas.0708986104, 2007.

Pisso, I., Sollum, E., Grythe, H., Kristiansen, N. I., Cassiani, M., Eckhardt, S., Arnold, D., Morton, D., Thompson, R. L., Groot Zwaaftink, C. D., Evangeliou, N., Sodemann, H., Haimberger, L., Henne, S., Brunner, D., Burkhart, J. F., Fouilloux, A., Brioude, J., Philipp, A., Seibert, P., and Stohl, A.: The Lagrangian particle dispersion model FLEXPART version 10.4, Geosci. Model Dev., 12, 4955-4997, https://doi.org/10.5194/gmd-12-4955-2019, 2019.

Ramachandran, R., Li, X., Movva, S., Graves, S., Greco, S., Emmitt, D., Terry, J., and Atlas, R.: Intelligent data thinning algorithm for earth system numerical model research and application, in: Proc. 21st Intl. Conf. on IIPS, 9-13 January 2005, San Diego, California, abstract number: 13.8, 2005.

Richman, M. B., Leslie, L. M., Trafalis, T. B., and Mansouri, H.: Data selection using support vector regression, Adv. Atmos. Sci., 32, 277-286, https://doi.org/10.1007/s00376-014-4072-9, 2015.

Rodgers, C. D.: Inverse Methods for Atmospheric Sounding: Theory and Practice, 4, Series On Atmospheric, Oceanic And Planetary Physics, World Scientific Publishing Company, London, 2000.

Schroeder, W. J., Zarge, J. A., and Lorensen, W. E.: Decimation of triangle meshes, in: PSIGGRAPH '92: Proceedings of the 19th annual conference on Computer graphics and interactive techniques, Chicago Illinois USA, 26-31 July 1992, 65-70, https://doi.org/10.1145/133994.134010, 1992.

Stein, A. F., Draxler, R. R., Rolph, G. D., Stunder, B. J. B., Cohen, M. D., and Ngan, F.: NOAA's HYSPLIT Atmospheric Transport and Dispersion Modeling System, B. Am. Meteorol. Soc., 96, 2059-2077, https://doi.org/10.1175/BAMS-D-14$00110.1,2016$.

Tadić, J. M., Qiu, X., Yadav, V., and Michalak, A. M.: Mapping of satellite Earth observations using moving window block kriging, Geosci. Model Dev., 8, 3311-3319, https://doi.org/10.5194/gmd8-3311-2015, 2015

Tadić, J. M., Qiu, X., Miller, S., and Michalak, A. M.: Spatio-temporal approach to moving window block kriging of satellite data v1.0, Geosci. Model Dev., 10, 709-720, https://doi.org/10.5194/gmd-10-709-2017, 2017.

Torres, A. D., Keppel-Aleks, G., Doney, S. C., Fendrock, M., Luis, K., De Maziere, M., Hase, F., Petri, C., Pollard, D. F., Roehl, C. M., Sussmann, R., Velazco, V. A., Warneke, T., and Wunch, D.: A Geostatistical Framework for Quantifying the Imprint of Mesoscale Atmospheric Transport on Satellite Trace Gas Retrievals, J. Geophys. Res.-Atmos., 124, 9773-9795, https://doi.org/10.1029/2018JD029933, 2019.

Wackernagel, H.: Multivariate Geostatistics: An Introduction with Applications, Springer, Berlin, 2003. 
Wu, D., Lin, J. C., Fasoli, B., Oda, T., Ye, X., Lauvaux, T., Yang, E. G., and Kort, E. A.: A Lagrangian approach towards extracting signals of urban $\mathrm{CO}_{2}$ emissions from satellite observations of atmospheric column $\mathrm{CO}_{2}\left(\mathrm{XCO}_{2}\right)$ : X-Stochastic TimeInverted Lagrangian Transport model ("X-STILT v1"), Geosci. Model Dev., 11, 4843-4871, https://doi.org/10.5194/gmd-114843-2018, 2018.
Zeng, Z., Lei, L., Hou, S., Ru, F., Guan, X., and Zhang, B.: A Regional Gap-Filling Method Based on Spatiotemporal Variogram Model of $\mathrm{CO}_{2}$ Columns, IEEE T. Geosci. Remote, 52, 3594 3603, https://doi.org/10.1109/TGRS.2013.2273807, 2014. 